\title{
Sustained-release niacin induced acute liver failure: an autopsy report
}

\author{
Hamza Tariq*, Francis E. Sharkey
}

Department of Pathology and Laboratory Medicine, University of Texas Health Science Center, 7703 Floyd Curl Dr, San Antonio, Texas, USA, 78229

Received: 21 December 2017 Accepted: 29 January 2018

\section{*Correspondence to: \\ Dr. Hamza Tariq, Email: TariqH@uthscsa.edu}

Copyright: (C) the author(s), publisher and licensee Medip Academy. This is an openaccess article distributed under the terms of the Creative Commons Attribution NonCommercial License, which permits unrestricted noncommercial use, distribution, and reproduction in any medium, provided the original work is properly cited.

\begin{abstract}
Niacin, either alone or in combination with other anti-hyperlipidemic agents, safely and effectively addresses most lipid abnormalities in patients with mixed dyslipidemias. Niacin is the only available agent that significantly lowers lipoprotein (a) and has the greatest high density lipoprotein cholesterol-raising effects of all available agents. Despite niacin's numerous beneficial lipid effects, patient compliance to long-term therapy is challenged by its common side effects which include nausea, pruritus, and vasodilatory flushing. The incidence of these unpleasant side effects in patients taking the Immediate Release (IR) form of the drug is close to $100 \%$. To avoid these side effects, Sustained Release (SR) formulations of the drug were created which lower the rate of nausea, flushing and pruritus markedly. Unfortunately, the SR form is associated with a high incidence of chemical hepatitis and rarely fulminant hepatic failure, which is not seen in patients taking the IR form. We report the autopsy findings of a 68 years old man who died of fulminant liver failure three weeks after switching from IR to SR form of niacin. All other toxic, infectious and autoimmune causes of liver failure were ruled out clinically. His liver biopsy one-week antemortem was consistent with chemical hepatitis, such as has been described for slow-release niacin. At autopsy the liver showed diffuse massive hepatic necrosis with no background fibrosis. SR Niacin is widely available over the counter; however, there is substantial scientific evidence that the drug is associated with potentially fatal hepatotoxicity.
\end{abstract}

Keywords: Niacin, Flushing, Hepatotoxicity, Sustained release

\section{INTRODUCTION}

Niacin was first recognized as an effective antihyperlipidemic and cholesterol lowering agent in $1955 .{ }^{1}$ Over time, the drug has evolved into a relatively inexpensive alternative to other lipid lowering drugs and currently is the most effective clinically available agent for increasing high-density lipoprotein cholesterol (HDL-C) levels. Due to these benefits, the drug has been extensively advertised and is sold over the counter in a variety of preparations, including crystalline or Immediate Release (IR), Sustained Release (SR), and Extended Release (ER) forms. Common side effects of niacin include nausea, fatigue, pruritus and flushing, with flushing being the major dose-limiting side effect. These side effects are more commonly observed with the IR form of the drug which is why many patients switch to the SR form. Unfortunately, the SR form is associated with a higher rate of hepatotoxicity. ${ }^{2}$ In a comparative study, approximately $50 \%$ of the patients receiving SR niacin experienced hepatotoxicity, especially with doses $>2,000 \mathrm{mg} /$ day, compared to none in the IR niacin group. ${ }^{3}$ In addition to reversible hepatotoxicity, rare cases of fulminant liver failure have been reported with the SR form. ${ }^{4-9}$

\section{CASE REPORT}

The patient was a 68 years old man with a past medical history of rheumatic fever, aortic stenosis and atrial fibrillation. He presented in the outpatient clinic three 
weeks before his death with sudden onset of jaundice which he noticed after switching from 2 grams of IR to 500 $\mathrm{mg}$ of SR niacin. The patient had been on 2 grams of IR niacin per day ( 4 doses of $500 \mathrm{mg}$ ) for his dyslipidemia but decided to switch to over the counter SR to avoid the unpleasant side effects and multiple doses per day. Three days later, he presented to the emergency room with worsening jaundice, weakness, tremors and confusion. Physical examination was significant for marked icterus and easy bruising of the skin. Upon initial evaluation, he was found to have acute liver failure with laboratory values shown in Table 1.

Table 1: Liver function tests at presentation.

\begin{tabular}{|c|c|c|}
\hline Lab result & Value & $\begin{array}{l}\text { Reference } \\
\text { range }\end{array}$ \\
\hline Total bilirubin & $36.0 \mathrm{mg} / \mathrm{dl}$ & $0.2-1.2 \mathrm{mg} / \mathrm{dl}$ \\
\hline Direct bilirubin & $22.3 \mathrm{mg} / \mathrm{dl}$ & $0-0.4 \mathrm{mg} / \mathrm{dl}$ \\
\hline $\begin{array}{l}\text { Aspartate } \\
\text { aminotransferase } \\
\text { (AST) }\end{array}$ & $502 \mathrm{U} / \mathrm{L}$ & $<32 \mathrm{U} / \mathrm{L}$ \\
\hline $\begin{array}{l}\text { Alanine } \\
\text { aminotransferase } \\
(\mathrm{ALT})\end{array}$ & $270 \mathrm{U} / \mathrm{L}$ & $<36 \mathrm{U} / \mathrm{L}$ \\
\hline $\begin{array}{l}\text { Alkaline phosphatase } \\
\text { (ALP) }\end{array}$ & 185U/L & 45-117U/L \\
\hline INR & 2.6 & $0.8-1.2$ \\
\hline Serum albumin & $2.5 \mathrm{~g} / \mathrm{dl}$ & $3.2-5.0 \mathrm{~g} / \mathrm{dl}$ \\
\hline Serum ammonia & $68 \mathrm{mcmol} / \mathrm{L}$ & $11-32 \mathrm{mcmol} / \mathrm{L}$ \\
\hline $\begin{array}{l}\text { Gamma } \\
\text { glutamyltransferase } \\
\text { (GGT) }\end{array}$ & $33 \mathrm{U} / \mathrm{L}$ & $9-48 \mathrm{U} / \mathrm{L}$ \\
\hline
\end{tabular}

There was no history of alcohol use, acetaminophen use, supplement intake, or other acute illness before the onset of the above described symptoms. Hepatitis B surface antigen, Hepatitis B core IgM, Hepatitis delta antibody, Hepatitis A IgM, Hepatitis E IgM and Hepatitis C antibody were negative. Liver-Kidney Microsomal, AntiMitochondrial, Anti-Smooth Muscle and Anti-Nuclear antibodies were undetectable. Serum ceruloplasmin, alpha- 1 antitrypsin and serum acetaminophen were within the reference ranges. His outpatient drugs included fish oil, glucosamine-chondroiton, melatonin, protonix, aspirin, lisinopril, SR-Niacin and tramadol.

Over the next few days his condition deteriorated, and he developed massive ascites and hepatorenal syndrome. Core biopsy of the liver showed marked cholestasis and portal tract expansion by a diffuse mononuclear cell infiltrate consisting of mixed lymphocytes, along with many neutrophils and a few histiocytes. Ductular proliferation was present multifocally in portal tracts. The lobular parenchyma displayed intralobular neutrophilic infiltration and marked disarray, with confluent portalportal and portal-centrilobular collapse. There was no background fibrosis (Figures 1 and 2). These findings were consistent with drug toxicity, such as has been described for SR niacin. A psychosocial evaluation was completed that denied the patient for transplant listing due to lack of viable caregiver support. He was placed in a skilled nursing home for comfort care where he died six days later.

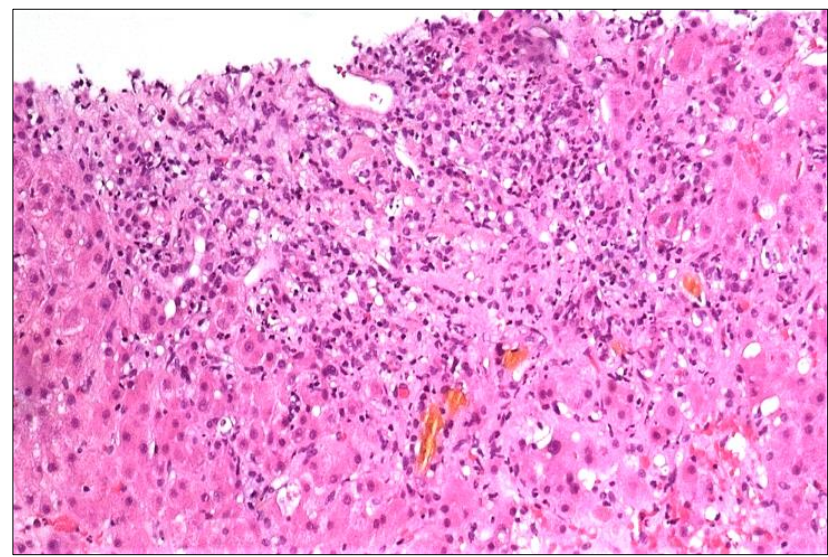

Figure 1: Core biopsy of the liver showing cholestasis and marked portal tract expansion by neutrophils, lymphocytes and histiocytes.

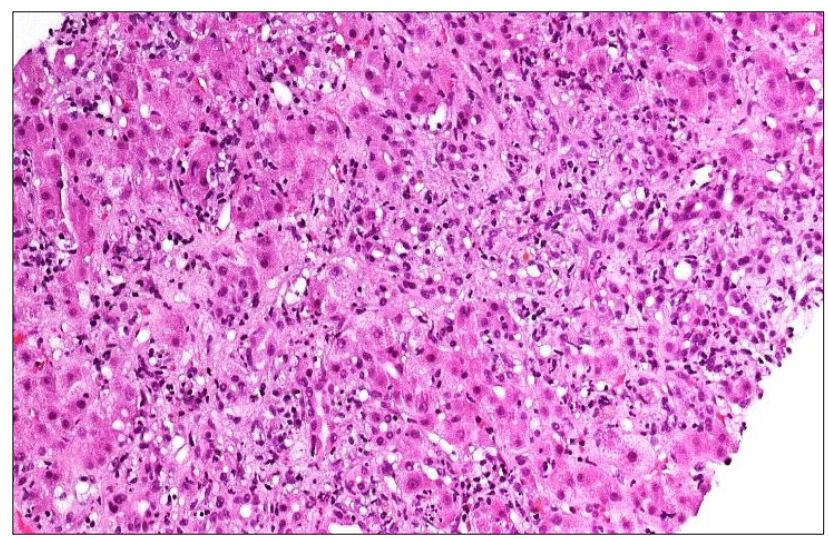

Figure 2: Core biopsy of the liver showing intralobular neutrophilic and lymphocytic infiltration and marked disarray of lobular architecture.

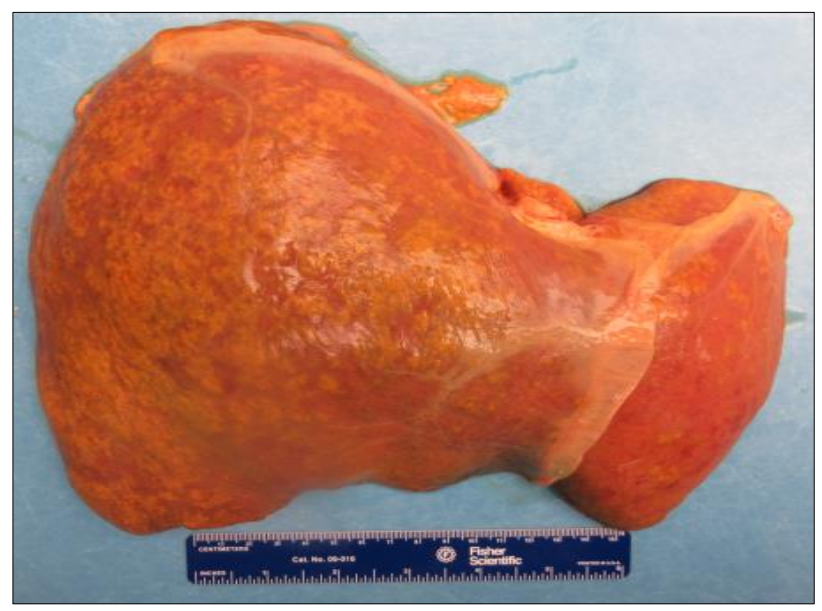

Figure 3: Liver at autopsy showing multifocal lobular necrosis. 
On autopsy exam, the patient exhibited several stigmata of acute liver failure including deeply jaundiced skin, bruising, scleral icterus and massive ascites. The liver was small and shrunken (weight: 1,100 grams) but did not show any evidence of fibrosis or cirrhosis. The capsular and cut surfaces showed extensive small yellow foci of necrosis and cholestasis (Figure 3). Microscopic examination of the liver confirmed the diffuse massive hepatic necrosis with almost complete loss of hepatocytes (Figure 4). Trichrome stain showed extensive areas of collapse with very limited fibrosis which favoured an acute/subacute process vs chronic injury.

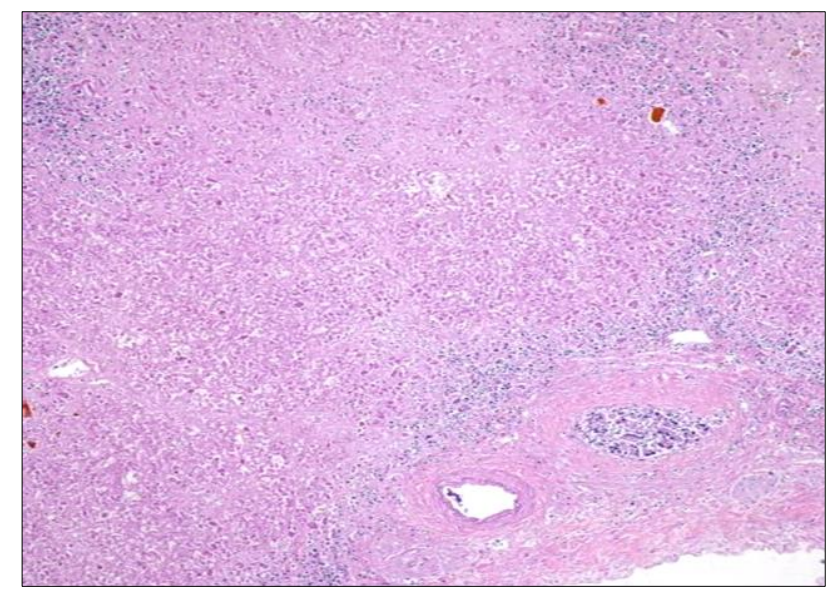

Figure 4: Microscopic section of the liver at autopsy showing massive hepatic necrosis, cholestasis and minimal fibrosis.

\section{DISCUSSION}

Niacin, either alone or in combination with other antihyperlipidemic agents, safely and effectively addresses most lipid abnormalities in patients with mixed dyslipidaemia. Niacin is the only anti-hyperlipidemic agent that has favorable effects on the complete lipoprotein profile. It significantly reduces levels of total cholesterol (TC), LDL cholesterol, and TG. Furthermore, niacin is the only available agent that significantly lowers lipoprotein (a) and has the greatest HDL cholesterol-raising effects of all available agents. Despite niacin's numerous beneficial lipid effects, patient compliance to long-term therapy is challenged by its common side effects including nausea, fatigue, pruritus, impaired glucose tolerance and prostaglandin mediated vasodilatory flushing. Multiple studies have shown flushing to be the major reason for discontinuation of niacin. Flushing is directly related to the rate of absorption of niacin, with a higher rate of absorption associated with a higher rate of flushing. In addition, flushing persists as long as plasma niacin levels are rising but abates when constant plasma niacin levels are reached. Crystalline or immediate-release (IR) niacin is rapidly absorbed by the body, and peak blood levels can be reached in as quickly as 30-60 min which is why the incidence of flushing among patients taking IR niacin is close to $100 \%$. To reduce these unpleasant side effects,
Sustained-Release (SR) niacin formulations were created to delay niacin absorption during treatment. Although SR niacin decreases flushing, it can cause hepatotoxicity and rarely fatal fulminant hepatic failure as in this patient. ${ }^{10}$

A comparative study showed that $52 \%$ of the patients on SR form of niacin developed some form of hepatotoxicity as compared to the IR form which did not cause hepatotoxicity in any patient. Although chemical hepatitis has been described with regular IR niacin, it occurs only at doses of $\geq 3 \mathrm{~g} /$ day. On the other hand, the SR form has been known to cause liver damage at doses as low as 500mg. ${ }^{11}$ The onset of hepatotoxicity generally appears anywhere from 1 week to 48 months after the initiation of the drug, and usually subsides with discontinuation. Irreversible fulminant hepatic failure has been reported but is very rare.

The possibility of toxicity from another of the patient's drugs has been considered. However, of these drugs, lisinopril has been known to cause mild elevation of transaminases but no cases of liver failure have ever been reported, whereas tramadol causes acute liver failure only in overdoses. The drug with the highest potential for hepatic injury was niacin, particularly the SR formulation.

The mechanism by which the SL form causes liver damage can be explained by understanding the metabolism pathways of the drug. The IR form of niacin uses a lowaffinity and high-capacity conjugation pathway that leads to prostaglandin-mediated vasodilation and subsequent cutaneous flushing. The SR form is metabolized via a high-affinity and low-capacity amidation pathway that avoids the synthesis of vasodilatory mediators but forms hepatotoxic nicotinomide and pyrimidine metabolites. The rapidly-released IR formulation overwhelms the higher affinity amidation pathway and the majority is metabolized using the high-capacity conjugation pathway, leading to a much lower rate of hepatotoxicity. ${ }^{12}$

The pattern of liver injury described for niacin is primarily hepatocellular, although cases with cholestatic and mixed patterns have been described. When the injury is the result of switching from the crystalline to the sustained release form, the injury may present acutely within days or a few weeks with a prodromal period of nausea, vomiting and abdominal pain, that is followed by jaundice and pruritus. Typically, serum aminotransferase levels are very high early during the injury and then usually fall rapidly with discontinuation or dose lowering. The clinical phenotype resembles acute hepatic necrosis, suggesting a direct toxic effect. The finding that niacin can be restarted at lower doses after an episode of clinically apparent injury indicates that the hepatic damage is unlikely to be idiosyncratic or due to hypersensitivity. ${ }^{13}$

The availability of niacin as an over-the-counter supplement makes it readily available to patients with no oversight of its use by a physician. In addition, there have been concerns raised over the use of supplemental preparations of niacin, as such products have less stringent 
US Food and Drug Administration regulations. ${ }^{3}$ This autopsy report reinforces the already existing scientific evidence that SR-niacin use is associated with potentially fatal hepatotoxicity; hence it should only be used under the supervision of a physician.

Funding: No funding sources Conflict of interest: None declared

Ethical approval: Not required

\section{REFERENCES}

1. Guyton J, Campbell K, Lakey W. Niacin: Risk Benefits and Role in Treating Dyslipidemias. Dyslipidemias. 2015;439-52.

2. Daul A, Beuhler M. Niacin Toxicity Resulting from Urine Drug Test Evasion. J Emerg Med. 2011;41:658.

3. Backes JM, Padley RJ, Moriarty PM. Important considerations for treatment with dietary supplement versus prescription niacin products. Postgrad Med. 2011;123:70-83.

4. Dalton TA, Berry RS. Hepatotoxicity associated with sustained-release niacin. Am J Med. 1992;93:102-4.

5. Fischer DJ, Knight LL, Vestal RE. Fulminant hepatic failure following low-dose sustained-release niacin therapy in hospital. West J Med. 1991;155:410-2.

6. Mullin GE, Greenson JK, Mitchell MC. Fulminant hepatic failure after ingestion of sustained-release nicotinic acid. Ann Intern Med. 1989;11:253-5.
7. Henkin Y, Johnson KC, Segrest JP. Rechallenge with crystalline niacin after drug-induced hepatitis from sustained-release niacin. JAMA. 1990;264:241-3.

8. Hodis HN. Acute hepatic failure associated with the use of low-dose sustained release niacin. JAMA. 1990;264:181.

9. Eswaran S, Alvey N, Fayek S, Shah N, Chan E. Niacin, the Internet, and Urine Drug Testing: A Cause of Acute Liver Failure. J Clin Toxicol. 2013;3:167.

10. Kamanna VS, Ganji SH, Kashyap ML. The mechanism and mitigation of niacin-induced flushing. International Journal of Clinical Practice. 2009;639:1369-77.

11. McKenney JM, Proctor JD, Harris S, Chinchili VM. A comparison of the efficacy and toxic effects of sustained- vs immediate-release niacin in hypercholesterolemic patients. JAMA. 1994;271:6727 .

12. Bhardwaj SS, Chalasani N. Lipid Lowering Agents That Cause Drug-Induced Hepatotoxicity. Clinics in liver disease. 2007;11:597.

13. LiverTox. Clinical and research information on drug induced liver injury, 2017. Available at: https://livertox.nih.gov/Niacin.htm\#structure.

Cite this article as: Tariq H, Sharkey FE. Sustainedrelease niacin induced acute liver failure: an autopsy report. Int J Basic Clin Pharmacol 2018;7:569-72. 